\title{
Sectional automatic adjustment of catalyst layers in gas and liquid phase reactors
}

\author{
Nikolay Merentsov ${ }^{1, *}$, Alexander Persidskiy ${ }^{2}$, Mikhail Topilin $^{3}$ and \\ Alexander Golovanchikov ${ }^{1}$ \\ ${ }^{1}$ Volgograd State Technical University, 400005 Volgograd, Russia \\ 2 JSC Federal Scientific and Production Centre «Titan - Barricady», 400071 Volgograd, Russia \\ ${ }^{3}$ Branch of LUKOIL-Engineering VolgogradNIPImorneft, 400078 Volgograd, Russia
}

\begin{abstract}
The paper provides a new approach to the high-quality implementation of gas-liquid and catalytic gas-and liquid-phase reactions in displacement reactors. The authors have described the scheme and algorithm for automatic control of the parameters of the catalyst layer. The authors have developed algorithms (mode) for automatic adjustment of the hydrodynamic and thermal modes of the catalytic section and also the principle of automatic adjustment of the system to a gradual or impulse adjusting mode which in case of liquid-phase reaction products results in active dispersion and mutual mixing of reaction products and sharp activation of hydrodynamic and diffusion processes. The article also covers the main requirements for elastically deformable catalytic layers and the advantages of using metalworking machines wastes as adjustable catalyst layers which will have a very significant environmental effect within the recycling and remarketing program.
\end{abstract}

\section{Introduction}

Mechanical, hydromechanical, heat and mass transfer processes traditionally belong to the main processes and apparatuses of chemical technologies, while chemical processes and devices in which they take place are distinguished as a special group in which, in addition to the above processes, complex processes of transformation of raw components into reaction products take place. We will dwell on chemical reactors having a wide range of design and mode features which depend on the conditions of the reactions, the rheological characteristics of the reaction masses, the variety of chemical reactions and the methods of influence on the reaction behaviour [1-14]. Displacement reactors are devices with an elongated body (channel) providing directional movement and mutual mixing of the reaction mass. There are many traditional factors and methods of influencing the process such as the hydrodynamic situation in the reactor, the thermal conditions of the chemical process, the conditions of heat exchange, the presence of a catalyst, the method of organizing the process, changing of the processes over time, the design features of the apparatus, etc [15-30]. The most promising areas of intensification of hydrodynamic and

\footnotetext{
*Corresponding author: steeple@mail.ru
} 
diffusion processes, as well as activation of reactions and influence on the conversion degree of reaction products are considered by the authors to be the ability to generate certain frequency radiations at the micro level contributing to the resonant reactions and the highest degree of energy transfer, and the creation of a developed flow mode and close to ideal flow structures of reaction masses at the macro level which may be accompanied by impulse-vibration modes of oscillations of the catalyst layers with the subsequent energy transfer from the catalyst to the reaction products which results in a sharp activation of the mutual mixing of the reaction products and prevents the catalyst layers from coking. The authors describe in detail the method of sectional automatic adjustment of the catalyst layers increasing the quality of work and control of the hydrodynamic modes of gas and liquid phase catalytic reactions (at the macro level). Automatic sectional adjustment of catalyst layers allows adjusting the system with the high-precision and influencing the chemical reactions directly in the process. To implement automatic adjustment catalysts are required to have volume elastic properties [31-34]. In addition, the catalyst should provide a developed contact surface with reaction products, and the reaction mass, in turn, should actively wash the surface of the catalyst giving an impulse to a chemical reaction. All these requirements are accumulated in the catalysts layers in the form of pressed sections of metal shavings (metalworking machine wastes) [31-41]. Moreover, the shaving materials themselves can be catalysts or catalysts can be applied to their developed ornate surface. The wastes of high-alloyed steels machining with high rates of corrosion resistance, acid resistance, heat resistance, heat resistance, etc. are mainly of interest. But the wastes generated during the machining of low-alloyed and carbon steel grades can also be applied in industry, for example, as catalyst layers of acetylene chlorination in bubbling reactors of production lines for producing technical solvents, etc.

The common positive properties possessed by metalworking machine wastes are an ornate structure, a very large specific surface area, the presence of cutting edges (micro ribs) leading to dispersion and playing an important intensifying role in the impulse vibration mode of the section, and volume elastic properties that allow performing automatic current sectional adjustment of catalyst layers, that means it gives us unique possibilities to control the reaction with the help of thermal modes and with the help of controlling the flows structures [42-44] and applying intensifying effects. Not to mention the fact that we are talking about the disposal of industrial wastes at metalworking machine-building enterprises which is very important from the point of view of the environmental safety.

\section{Methods and materials}

The main task of the automatic adjustment system of a catalytic reactor is to specify the optimal mode and maintain the reactor operating parameters corresponding to the given final concentration of reaction products (conversion degree) while ensuring stable, safe operating conditions. Figure 1 shows a section of a catalytic or gas-liquid bubbling reactor, which can be automatically adjusted by hydraulic cylinders 1 , which gradually or impulsively move the movable grid 2 setting a limit to the layer of the elastically deformable catalyst layer 3 . The section is packed in a heat-exchange jacket 4 , which makes it possible to partially control the thermal mode during the implementation of nonisothermal catalytic or gas-liquid reactions. 


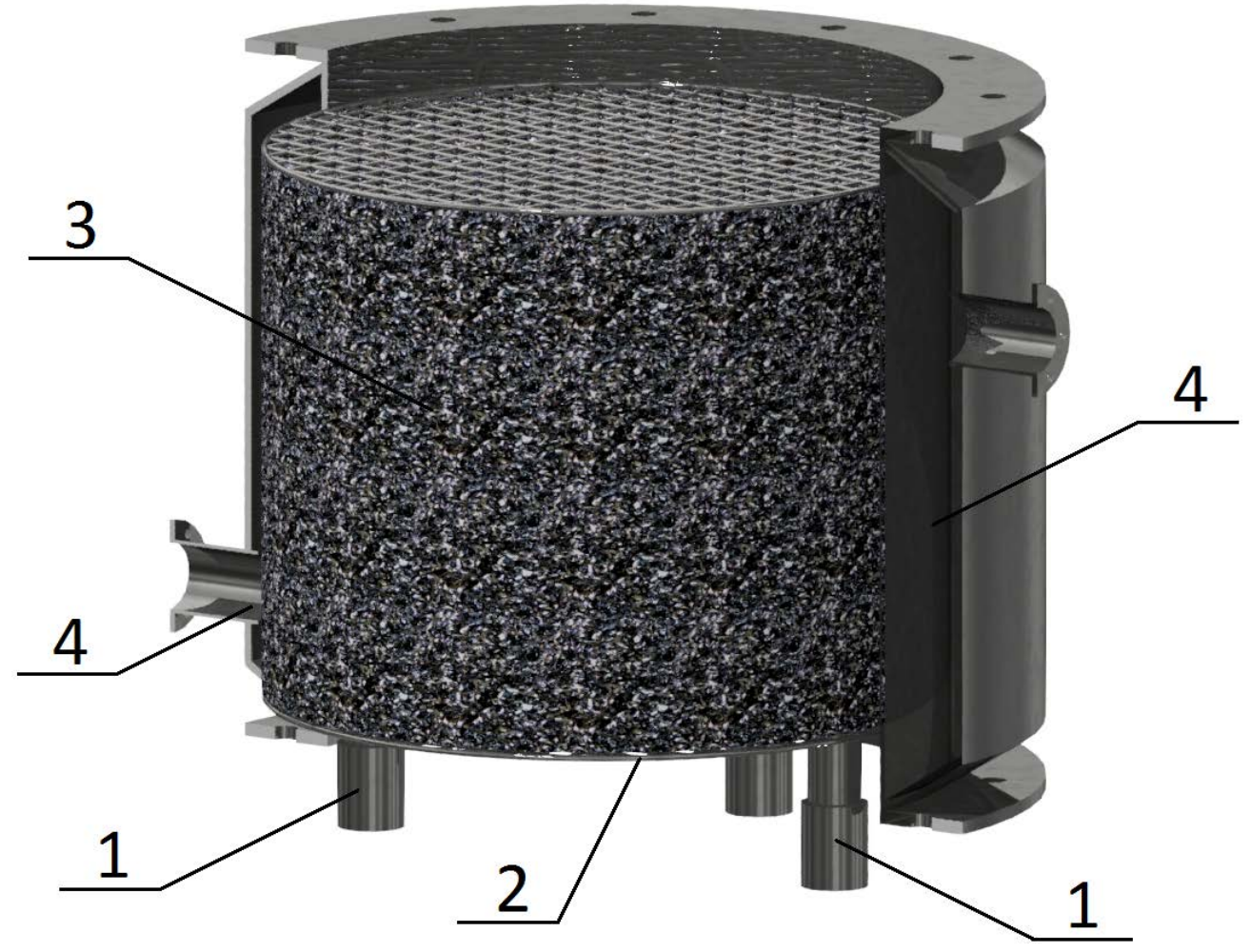

Fig. 1. Automatically adjustable section of the catalytic or gas-liquid reactor.

Figure 2 shows a scheme of a catalytic reactor section and an automated mode control system. The section consists of an elastically deformable catalyst layer (metal shavings) laid between two gratings. The upper (lower) grid $U G(L G)$ is driven by the hydraulic cylinders $H C 1$ and $H C 2$. Due to the oil pressure, the hydraulic cylinders lower the grid and compress the catalyst layer. The grid is returned to the upper position due to forcing oil into the lower chambers of the cylinders, and the elasticity of the catalytic layers. The ultrasonic sensor $S 2$ mounted on the body of one of the hydraulic cylinders determines the distance to the target $\mathrm{T}$, a metal plate mounted on a bracket on the upper grid. Thus, according to the reading of the sensor $S 2$ you can judge the position of the grid. Below the catalyst layer the sensor $S 3$ is installed, it measures the concentration of the reaction products. The type and design of this sensor are determined by the substance to be extracted. An oil volumetric flow sensor $S 1$ is installed in the hydraulic line supplying oil to the upper chambers of the hydraulic cylinders. All sensors transmit the digital data to a programmable logic controller $P L C$. The controller controls the hydraulic valves by closing the discrete contacts and giving digital commands, and valves V2, V3, V4, V5 have an electromagnetic drive and are controlled by discrete signals, and valve $V 1$ is driven by a stepping motor and allows controlling the flow section of the hydraulic line. Valves V2 and V3 control the injection of oil into the upper and lower chambers of the hydraulic cylinders, respectively, and V4 and V5 serve to dump oil from the cylinder chambers into the oil reservoir OR. Forcing oil pressure is provided by the oil pump $P$ driven by an electric motor $M$. Hydraulic accumulators $A 1$ and $A 2$ smooth out pressure surges in the line when opening valves $V 2$ and $V 3$, and check valves $V O 1$ and $V O 2$ prevent oil from moving towards the pump. 


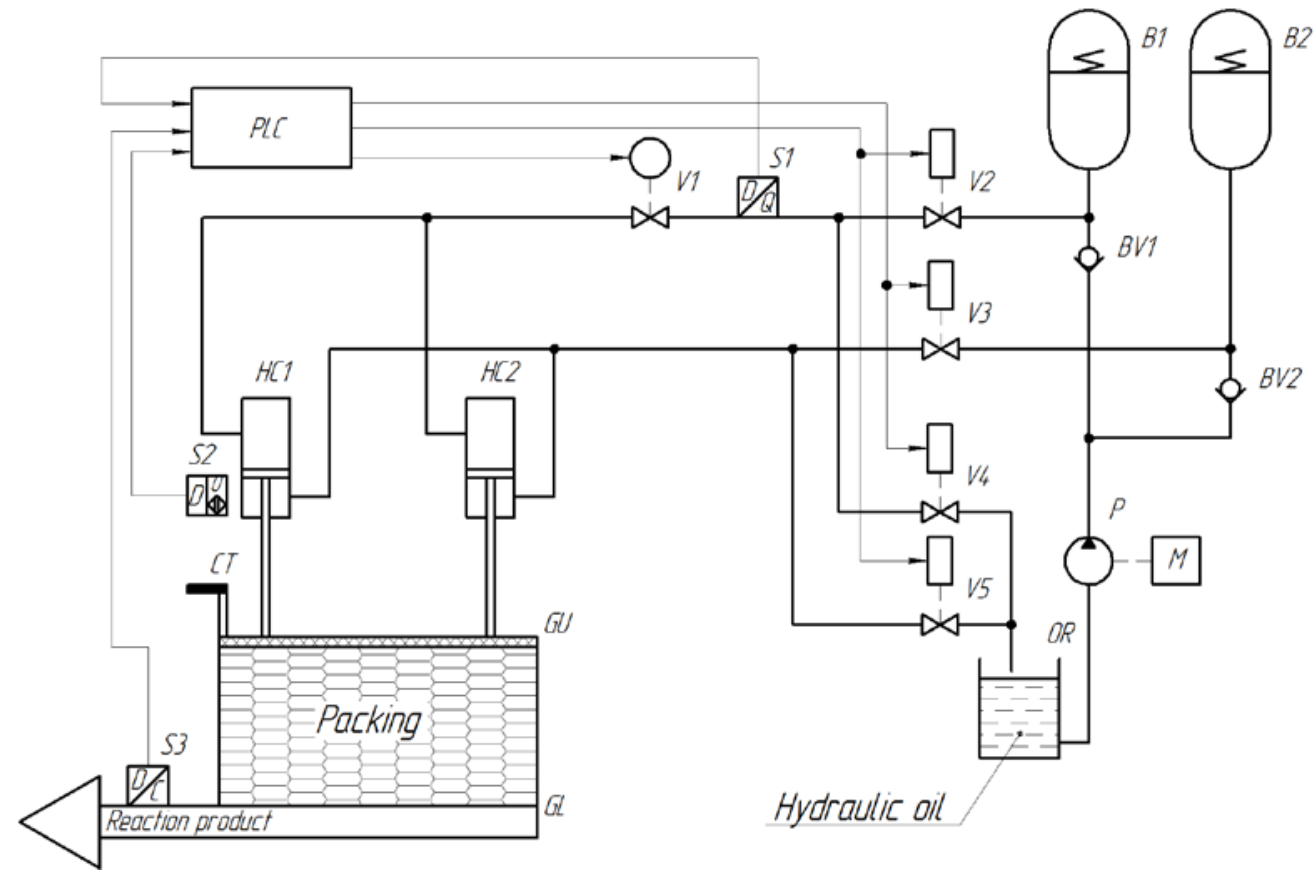

Fig. 2. Scheme of automatic control of the catalytic reactor section.

The control program the algorithm of which is shown in Figure 3s cyclically executed in the PLC. The program operates as follows. Before starting the extractor, the operator enters the following parameters: amplitude $A$ of the pulsation of the elastically deformable catalyst layer as a percentage of the maximum possible, initial frequency of the pulsation F0 which starts automatic selection of the optimal frequency, frequency adjustment step $N$ which determines the accuracy of the optimal pulsation frequency, necessary concentration of the reaction product Copt and the accuracy of determining the concentration of Acc, which shows the maximum allowable deviation of the concentration from the optimum.

Next, the parameters set by the operator make the program determine the lower limit that the grid should reach in the pulsation mode. Then it determines the step by which the grid position is changed during adjustment in the static mode.

From sensor V3 the program receives the value of the actual concentration of the reaction product at the exit from the section, and then calculates the lower and upper limits of the acceptable concentration of the reaction products.

After that, the program checks whether the actual concentration of the reaction products is less than the minimum acceptable. If it is less, the program checks in which mode the reactor is currently operating. If the value of the Pulse variable is 0 , it is the static mode, if the value of the Pulse variable is 1 , it is the pulsation mode. In the static mode, the grid constantly compresses the catalytic unit to a level corresponding to the given concentration of the catalysis product at the exit of the section. In the pulsation mode, the grid reciprocates with the given amplitude and frequency corresponding to the given concentration of reaction products (conversion degree).

In the static mode, the controller gives a command to open valve $V 1$ by half of its capacity which provides gradual movement of the grid during adjustment, and then increases by one step the value of the variable Lvlopt, which determines the optimal position of the grid providing the given product concentration, i.e. initiates its lowering by one step. 


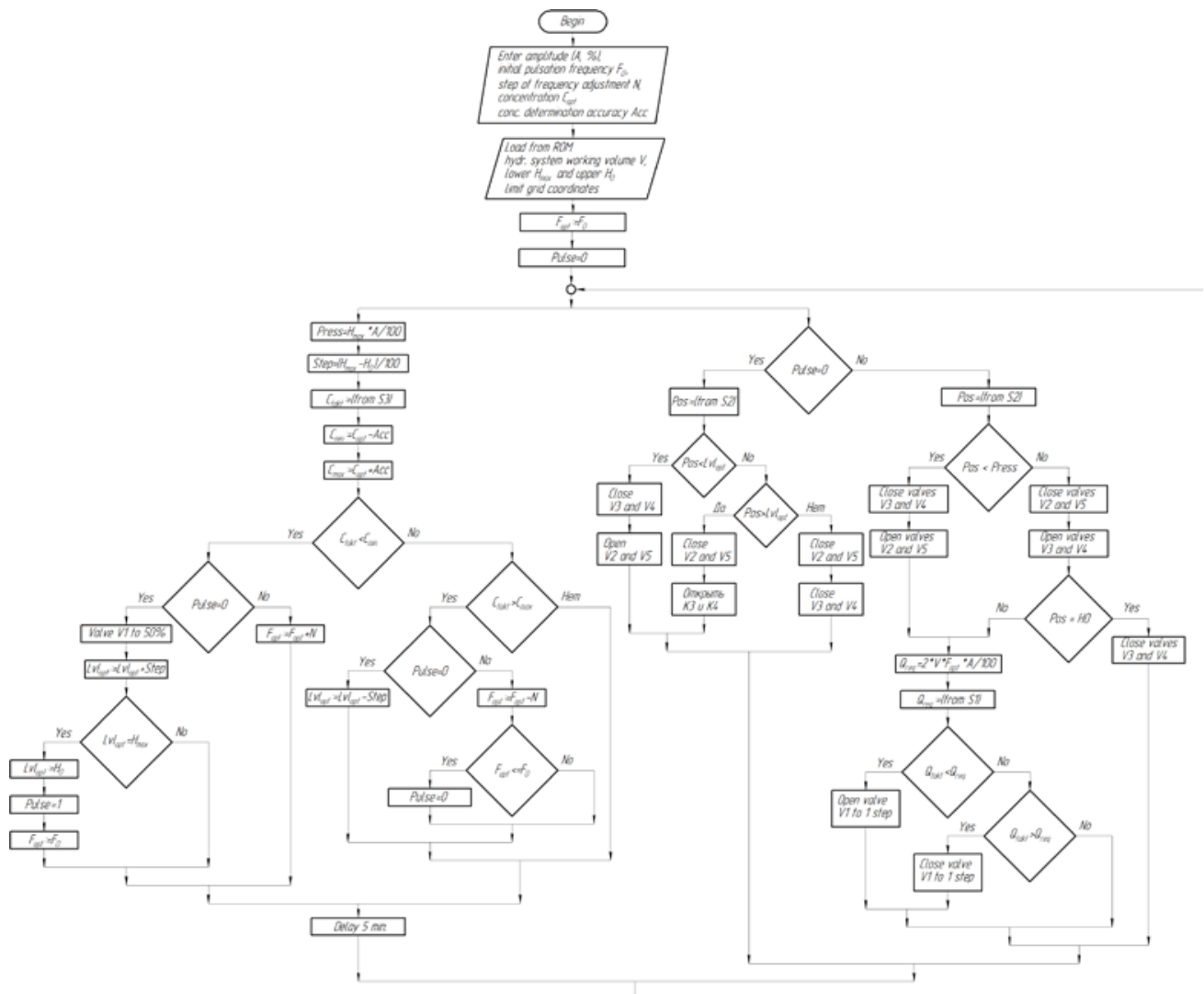

Fig. 3. Algorithm of automatic control of the catalytic reactor section.

Then the program checks whether the value of the optimal position of the grid has reached the lower limit of the grid mobility Hmax. If it has, the program switches the operating mode of the reactor to pulsation.

If the mode of operation was pulsation, the program increases the value of the pulsation frequency of the catalyst layer Fopt by 1 (one) step.

If the actual concentration of the reaction products Sfact is greater than the acceptable Cmax, the program in the static operation mode reduces the value of the variable determining the optimal position of the grid Fopt, i.e. initiates its rise by one step. In case of the pulsation mode, the program reduces the pulsation frequency of the $t$ unit Fopt by one step, and then checks whether the specified pulsation frequency is less the minimum allowed. If it is, the program switches the reactor to the static mode.

After that, the execution of this algorithm branch is interrupted for 5 minutes.

In parallel with these actions, an algorithm is performed that organizes the movement of the catalyst layer in accordance with the specific mode.

The program checks which mode of reactor operation is set. In the static mode, the program receives from the sensor $S 2$ the current coordinate of the grid Pos, and then compares it with the coordinate value Lvlopt calculated earlier. If the value of Pos is less than Lvlopt, the grid is above the optimal position, and the packing must be compressed. The controller gives a command to close valves V3 and V4, and then to open valves V2 and $V 5$. This causes oil to be pumped into the upper chambers of the hydraulic cylinders and the grid is lowered and the catalytic unit is compressed. If the value of Pos is greater than Lvlopt, the grid is below the optimal position, and the catalyst layer must be released. The 
controller gives a command to close valves V2 and V5, and then to open valves V3 and V4. The grid rises, and the catalytic unit is released.

If the value of Pos is equal to Lvlopt, all valves are closed, the grid stops and is fixed.

In the pulsation mode, the program reads the current grid coordinate from the sensor $S 2$. If the grid coordinate is less than the required one, the controller gives a command to close the V3 and V4 valves and open the V2 and V5 valves. The oil is injected into the upper chambers of the cylinders and dumped from the lower ones and the grid begins to move downward compressing the catalyst layer. If the grid coordinate is greater than the required one, the controller gives a command to close the V2 and V5 valves and open the V3 and V4. Oil begins to be pumped into the lower chambers of the cylinders and dumped from the upper ones and the grid begins to move upwards.

After that, the program calculates how much oil should be consumed in the line when it is pumped into the upper chambers of the hydraulic cylinders and dumped from them, receives actual data on oil consumption from the $S 1$ sensor and compares them with the required value. If the actual oil consumption is less than the required one, the controller commands the valve drive $V 1$ to open it by one step, if the flow is higher, close it by one step. If it is equal, do nothing. As a result, the more the valve $V 1$ is open, the faster the oil fills the hydraulic cylinders and is dumped from them, hence the frequency of cyclic movements of the grid becomes higher.

When the grid reaches the top position, valves V3 and V4 close.

Thus, when cyclically performing this algorithm, the grid moves up and down, causing the catalyst layer of the material to pulsate.

In parallel, the program receives from the sensor S3 the value of the actual concentration of the reaction mass and compares it with the required value. If the concentration is less than the set concentration, the program increases the frequency of the pulsations of the packing by step $N$, if the concentration is greater, it decreases by the same step.

\section{Conclusions}

The developed scheme and algorithm for the automatic sectional adjustment of catalyst layers in the implementation of gas and liquid catalytic reactions and gas-liquid bubbling reactions allow quality control of the processes creating close to ideal structures of the reaction mass flows activating mutual mixing and dispersing the reaction products (for liquid-phase reaction products) in a pulsation mode. Automatic adjustment of hydrodynamic and diffusion parameters can be carried out in both static and dynamic pulsation modes which is especially important when conducting liquid-phase catalytic reactions. In addition to the hydrodynamic setting, thermal modes of operation can also be adjusted in the implementation of non-isothermal catalytic reactions.

The proposed chemical engineering direction implies a close relationship with machinebuilding and metal-working enterprises, since the use of elastically deformable packing units provides the effective use of industrial wastes of such enterprises which will undoubtedly have a significant environmental and energy-saving effect improving waste management systems.

\section{References}

1. L.S. Gordeev, A.N. Labutin, E.L.Gordeeva, Theor. Found. of Chem. Eng. 48, 637 (2014)

2. V.P. Meshalkin, V.V. Men'shikov, S.V. Panchenko, D.S. Panchenko, A.S. Kazak, 
Theor. Found. of Chem. Eng. 49, 606 (2015)

3. Y.V. Sharikov, F.Y. Sharikov, O.V. Titov, Theor. Found. of Chem. Eng. 50, 225 (2016)

4. E.A. Shulaeva, N.S. Shulaev, Chem. and Pet. Eng. 52, 3 (2016)

5. D.L. Astanovskii, L.Z. Astanovskii, Chem. and Pet. Eng. 41, 513 (2005)

6. M. Bakošová, A. Mészáros, J. Oravec, J. Klemeš, Theor. Found. of Chem. Eng. 46, 740 (2012)

7. S.V. Frolov, A.A. Tret'yakov, V.N. Nazarov, Theor. Found. of Chem. Eng. 40, 349 (2006)

8. A.A. Kazakov, A.M. Bessarabov, V.E. Trokhin, A.G. Vendilo, Chem. and Pet. Eng. 51, 597 (2016)

9. A.N. Labutin, V.Y. Nevinitsyn, Theor. Found. of Chem. Eng. 48, 296 (2014)

10. V.P. Meshalkin, S.V. Panchenko, M.I. Dli, D.S. Panchenko, Theor. Found. of Chem. Eng. 52, 166 (2018)

11. V.M. Shmelev, Theor. Found. of Chem. Eng. 40, 526 (2006)

12. V.M. Shmelev, V.M. Nikolaev, Theor. Found. of Chem. Eng. 42, 19 (2008)

13. V.M. Shmelev, V.M. Nikolaev, Theor. Found. of Chem. Eng. 43, 50 (2009)

14. M.G. Berengarten, A.O. Nevelson, A.S. Pushnov, Chem. and Pet. Eng. 48, 723 (2013)

15. I.V. Derevich, D.D. Galdina, V.S. Ermolaev, V.Z. Mordkovich, Theor. Found. of Chem. Eng. 46, 8 (2012)

16. V.N. Ivanets, G.E. Ivanets, A.N. Potapov, Chem. and Pet. Eng. 51, 221 (2015)

17. V.A. Kirillov, N.A. Kuzin, V.A. Kuz'min, V.B. Skomorokhov, A.B. Shigarov, Theor. Found. of Chem. Eng. 39407 (2005)

18. A.A. Kazakova, Y.V. Nikiforov, B.K. Zuev, Chem. and Pet. Eng. 51, 84 (2015)

19. K.B. Komissarov, A.V. Fil', V.A. Finochenko, Chem. and Pet. Eng. 53, 814 (2018)

20. A.G. Nagiev, S.A. Khalilov, A.S. Guseinova, G.A. Nagiev, Chem. and Pet. Eng. 52, 15 (2016)

21. L.A. Nikolaeva, M.A. Golubchikov, A.R. Minneyarova, Chem. and Pet. Eng. 53, 806 (2018)

22. E.V. Polienova, A.Y. Valdberg, Chem. and Pet. Eng. 47, 104 (2011)

23. S.G. Zavarukhin, G.G. Kuvshinov, Theor. Found. of Chem. Eng. 40, 519 (2006)

24. M.F. Rudenko, Yu.V. Chivilenko, V.I. Cherkasov, Chem. and Pet. Eng. 42, 445 (2006)

25. V.K. Leont'ev, A.V. Sugak, Yu.A. Moskvichev, E.V. Shalygin, P.A. Nikiforov Chem. and Pet. Eng. 44, 628 (2008)

26. A.V. Leont'ev, I.V. Galitskii, G.M. Goncharov, A.V. Sugak, Chem. and Pet. Eng. 41, 45 (2005)

27. S.L. Nazanskii, A.V. Solokhin, V.S. Timofeev, Theor. Found. of Chem. Eng. 43, 671 (2009)

28. Y.V. Nikiforov, V.B. Vorotyntsev, Chem. and Pet. Eng. 49, 314 (2013)

29. A.G. Tkachev, Chem. and Pet. Eng. 43295 (2007)

30. A.Y. Valdberg, N.E. Vaulin, A.V. Simnanskii, A.E. Antoshin, V.I. Lazarev Chem. and Pet. Eng. 46, 425 (2010)

31. N. Merentsov, A. Persidskiy, V. Lebedev, Materials Today: Proceedings (2019) https://doi.org/10.1016/j.matpr.2019.07.037 (to be published) 
32. N. Merentsov, A. Persidskiy, M. Topilin, Materials Today: Proceedings (2019) https://doi.org/10.1016/j.matpr.2019.07.039 (to be published)

33. N. Merentsov, A. Persidskiy, V. Lebedev, M. Topilin, A. Golovanchikov Advances in Intelligent Systems and Computing 983, 560 (2019)

34. A.V. Persidskiy, N.A. Merentsov, V.N. Lebedev, A.B. Golovanchikov, IOP Conf. Ser.: Earth and Environmental Science 288, 012110 (2019)

35. A.B. Golovanchikov, N.A. Merentsov, V.A. Balashov, Chem. and Pet. Eng. 48, 595 (2013)

36. N.A. Merentsov, V.A. Balashov, D.Y. Bunin, V.N. Lebedev, A.V. Persidskiy, M.V. Topilin, MATEC Web of Conf. 243, 5 (2018)

37. N. Merentsov, A. Persidskiy, V. Lebedev, N. Prokhorenko, A. Golovanchikov Advances in Intelligent Systems and Computing 983, 547 (2019)

38. A.B. Golovanchikov, V.A. Balashov, N.A. Merentsov, Chem. and Pet. Eng. 53, 10 (2017)

39. N.A. Merentsov, A.V. Persidskiy, M.V. Topilin, V.N. Lebedev, V.A. Balashov, A.B. Golovanchikov, Journal of Phys.: Conf. Ser. 1278, 012024 (2019)

40. A.B. Golovanchikov, N.A. Merentsov, M.V. Topilin, A.V. Persidskiy, IOP Conf. Ser.: Earth and Environmental Science 288, 012089 (2019)

41. N.A. Merentsov, V.N. Lebedev, A.V. Persidskiy, V.A. Balashov, IOP Conf. Ser.: Earth and Environmental Science 288, 012106 (2019)

42. A. Golovanchikov, N. Merentsov, Advances in Intelligent Systems and Computing 983, 635 (2019)

43. A. Golovanchikov, N. Merentsov, Advances in Intelligent Systems and Computing 983, 645 (2019)

44. A.B. Golovanchikov, N.A. Merentsov, M.V. Topilin, Journal of Phys.: Conf. Ser. 1278, 012023 (2019) 